\title{
MEMBERSHIP LIST
}

\section{British Columbia Division}

Dr J. L Andrews

1946 Mayfarr Dr

Victoria, B C

Dr Murray G, Atnıkov

$559 \mathrm{E} 12$ th Ave

New Westminster, B C

Dr G W Barnes

1731 Dublin St

New Westminster, B.C

Dr Manon Berry

c/o Essex

5989 Cartier St.

Vancouver 13, B C

Dr G B Bigelow

612 View St

Victoria, B C

Dr A C Blaur

$1009 \mathrm{~W}$ 33rd Ave

Vancouver, B.C

Dr William H Blaur

124 Wedgewood Ave

New Westminster, B C

Dr J J Carroll $1530 \mathrm{~W}$ 37th Ave

Vancouver, B C

Dr H C Carruthers

3067 Oakdowne

Victona, B C

Dr. Jone Chang

3237 Copley St

Vancouver 6, B C

Dr. I. M Clearhue

2445 Lansdowne Rd

Victoria, B C.

Dr F S Chu

$16 \mathrm{E}$ Hastungs $\mathrm{St}$

Vancouver 4, B.C

Dr. Gordon F. Clark

4220 Evergreen

West Vancouver, B C

Dr. Donald R Collins

Dept. of Andesthesia

Vancouver General Hospital

Vancouver, B.C
Dr. B. Cragg

2765 Sea View Rd.

Victoria, B.C.

Dr. Lois Grawford.

620 King George's Way

Park Royal P O'

Vancouver, B.C.

Dr Lois E Davies

829 West Broadway

Vancouver, B.C.

Dr George F. Day

3392 W 38th Ave.

Vancouver, B C.

Dr - ohn W Denuis

145c W 39th Ave.

Vancouver 13, B.C.

Dr A N Dobrey

5665 Granville St

Vancouver, B.C.

Dr William A Dodds

4789 Haggart St.

Vancouver, B C

Dr Alan A Drysdale

Assistant Resident in Anaesthesia

Vancouver General Hospital

Vancouver, B.C

Dr. C. W. Duck

2796 Dufferm

Victoria, B C.

Dr E O. DuVernet

9031 Hudson St.

Vancouver, B.C.

Dr Willıam L. Esdale

4487 Townley St

Vancouver, 8, B.C.

Dr R A Fortye

Royal Jublee Hospital

Victona, B.C.

Dr D. D. Freeze

1185 West 49th Ave.

Vancouver, B C.

Dr James B. Fulton

2315 W. 18th Ave

Vancouver, B.C. 
Dr Gordon . Fyffe

Dept of Patiology

The Deaconess Hospital

Spokane 4, Wash.

Dr A.K Gibbons

935 W. 19th Ave.

Vancouver, B C

Dr. James A Gibson

Surrey Memorial Hospital

P O Box 190, North Surrey, B C

Dr. H B Graves

6016 Alma Rd

Vancouver, B C

Dr John W Green

3096 Cadboro Bay Rd

Victona, B C

Dr Marion Gruffiths,

151 West Woodstock Ave,

Vancouver, B.C.

Dr Edwin R Hall

665 Farmile Rd

West Vancouver, B C

Dr. W M Hall

5511 Wallace

Vancouver, B C

Dr Murray C Hay

3724 Clark Dr

Vancouver 10, B.C.

Dr Edwon R Henry

2025 Lansdowne Rd

Victoria, B C

Dr J M Hiddleston

\#12, 3837 Impenal St

$S$ Burnaby, B C

Dr George R Horne

Shaughnessy Hospital

Vancouver, B C

Dr W S Huckvale

107 Ritchie Ave

Trall, B G.

Dr Peter W Hudson

4094 W. 33rd Ave

Vancouver, B C.

Dr Edward N Hughes

2995 West 34th Ave

Vancouver, B.C
Dr Jean Hugll

3855 Puget Dr.

Vancouver, B.C

Dr John A. Hutchison

612 View St.

Victoria, B C

Dr Lilıan Hutton

5475 Manitoba St

Vancouver, B.C.

Dr. Leonard C Jenkuns

Vancouver General Hospital

Vancouver, $\mathrm{B}$ C

Dr R A Jones

5694 Heather St

Vancouver, B C

Dr Erna Keddis

PO Box 132

Port Albern, B C.

Dr Patrick N Kenny

1492 W. 45th Ave

Vancouver 13, B C

Dr H A KesteJ

6476 Granville St

Vancouver, B C

Dr Barbłra $P$ Kraft

PO Box 310

Kitimat, B C.

Dr K W Langston

3280 Mathers Ave.

West Vancouver, B.C

Dr Michael Lattey

3009 31st Ave

Vernon, B C

Dr George H. Laycraft

Vancouver General Hospital

Vancouver, B C

Dr B C Leach

P O. Bor 448

Nanaimo, B.C

Dr R I Logan

Apt. 602, 511 Ash St

New Westminster, B C

Dr. John B H Lusk

Port Hammond, B C 
Dr. F. W McCaffrey

4094 W. 29th Ave

Vancouver 8, B C.

Dr Hugh H Macartney

100 Bonnymur Dr

West Vancouver, B C

Dr M Watson MacCrostıe

$3062 \mathrm{~S}$ W Marme Dr

Vancouver, B C

Dr Alexander D McDougall

Royal Inland Hospital

Kamloops, B C

Dr H B MacEwen

1335 West 55th Ave

Vancouver 14, B C

Dr John C McGregor

13032 104th Ave

North Surrey, B C

Dr Dorıs E MacKay

$1041541 \mathrm{~W}$ Broadway

Vancouver, B C

Dr Angus A MacMillan

2412 Edgar Cres

Vancouver $8, \mathrm{~B} \mathrm{C}$

Dr Norman McMillen

1961 Allison Rd

Vancouver, B C

Dr J A MacNab

2474 W 36th St

Vancouver, B C

Dr Willam G Markham

1937 West 44th Ave

Vancouver, B C.

Dr Marıa J Mate

1235 Comox

Vancouver 5, B C

Dr Donald Matheson

$2885 \mathrm{~W}$ 34th Ave

Vancouver 13, B.C

Dr Lorenzo Meloche

$4286 \mathrm{~W}$ 8th Ave

Vancouver, B C

Dr R D. Mullar

$2175 \mathrm{~W}$ 49th Ave

Vancouver, B C

Dr Irene Edith Miller

1275 W. 12th Ave

Vancouver, B C
Dr Mary Louse Miller 7061 Cypress St

Vancouver 14, B.C

Dr Robert S. Moir

108 West 19th Ave

Vancouver, B C.

Dr John E. Nixon

5515 Laburnum St

Vancouver 13, B C.

Dr J P O'Donnell

$4116 \mathrm{~W}$ 8th Ave

Vancouver, B C

Dr Thomas J O'Nell

1635 Abbott St

Kelowna, B.C

Dr John L Oulton

Dept of Anaesthesia

Vancouver General Hospital

Vancouyer, B C

Dr Peter B Percheson

$1570 \mathrm{~W}$ 36th Ave.

Vancouver 13, B C

Dr J C. Poole

1049 Devonshrre Cres

Vancouver, B C

Dr. I Porter

1645 Commercial

Vancouver, B C

Dr. Tom E Powell

Vancouver General Hospital

Vancouver, B C

Dr H S. Randall

$4786 \mathrm{~W}$ 2nd St

Vancouver, B C

Dr. Edward L Redpath

Internes' Quarters

Vancouver General Hospital

Vancouver, B C

Dr. D G Revell

1254 Judge Place

Victoria, BC

Dr. Elsie Ritch

3779 Blenheim St

Vancouver, B.C

Dr G L Robinson

$3725 \mathrm{~W}$ 29th Ave

Vancouver, B C

Dr. Alice K. Ronan

4509 West 5th Ave.

Vancouver 8, B.C. 
Dr. Colin A. Sands

4705 Chancellor Blvd

Vancouver 8, B.C.

Dr Maxwell H Schultz

563 Glenross

West Vancouver, B.C

Dr Guy Screech

3680 Cadboro Bay Rd.

Victoria, B.C

Dr. M B Shea

$1530 \mathrm{~W}$ 37th Ave.

Vancouver, B C.

Dr. Robert E. Simpson

$1281 \mathrm{~W}$ 32nd Ave.

vancouver, B C.

Dr G. E Sleath

3832 Forest St.

South Burnaby, B.C

Dr N. A Stewart

4149 Cypress

Vancouver, B C

Dr Robert James Stewart

1040 16th Ave W.

Vancouver 9, B C

Dr John R. Stone

3656 Crestview Rd.

Victona, B C

Dr Peter A Tannen

1702 Chesterfield Rd

North Vancouver, B C

Dr James V. Taylor

Internes' Residence

Vancouver General Hospital

Vancouver, B.C.

Alun Phllp Thomas

Irving Clinic

PO Box 150

Kamloops, B C
Dr N. M Thornton

\#11, 5781 Vine St

Vancouver, B C

Dr. Robert Hugh Thorp

690 Knockmaroon Rd

West Vaacouver, B.C

Dr Benjamin R. Townsley 4132 Vurgina Cres

North Vancouver, B C

Dr Donald A Walker

4376 Price Cres.

South Burnaby B C

Dr. Fred Walton

827 Cherry

New Westminster, B C

Dr Enc Webb

1512 Acadea Rd

Vancouver, B C

Dr Henr Wetselaar

I430 E 7th Ave

Vancouver, B C

Dr R G Whitehead

3850 Synod Rd

Victoria, B.C

Dr Floyd G Wilhams

$2810 \mathrm{~W}$; 31st Ave

Vancouver 8, $3 \mathrm{C}$

Dr. Ralph S Woodsworth

4638 Pune Cres

Vancouver, B C

Dr G H. Worsley

191 Cameron Rd.

New Westmmster, B C

Dr Maxwell Yates

2351 Lansdowne Rd

Victoria, B C

\section{Alberta Division}

Dr A Aeillo

Drumheller Associated Physicians

Drumheller, Alta.

Dr J A. Alton

Lamont, Alta
Dr J O Ancierson

406 Scarboro Ave

Calgary, Alta

Dr Donald F Cameron 9015 Saskatchewan Dr Edmonton, Alta 
Dr. J E Carmıchael

11033 80th Ave.

Edmonton, Alta.

Dr John B. Collip

11141 91st Ave.

Edmonton, Alta

Dr. Victor E Crapnell

Haig Clinic

Lethbridge, Alta

Dr Edgar Diamond 2326 3rd Ave N W.

Calgary, Alta.

Dr Enc H Dobbs

635 Britannia Dr. S W, Calgary, AJta

Dr William A. Doll

10281 Princess Elizabeth Ave

Edmonton, Alta

Dr Richard P. Douglas

39064 A St. S W.

Calgary, Alta

Dr C Denzil Elton

11627 78th Ave

Edmonton, Alta

Dr Fredenck C. Emberton

Newell Assocıate Clınic

Brooks, Aita

Dr Harold H. England

14616 88th Ave.

Edmonton, Alta.

Dr John F Farley

7143 Saskatchewan Dr.

Edmonton, Alta

Dr Frank F. Fish

201 33rd Ave. S.W.

Calgary, Alta.

Dr C M. Fletcher

8711 120th St.

Edmonton, Alta.

Dr. Norman E. Foster

117 Supenor Ave

Calgary, Alta

Dr. E A Gain

8958 Windsor Rd

Edmonton, Alta.

Dr Willam Hugh Graham

13733 115th Ave

Edmonton, Alta
Dr. A. S. Hall

Dept. of Anaesthesia

Royal Alexandra Hospit al

Edmonton, Alta

Dr. John R. Hemstock

11949 Jasper Ave., Apt. 6

Edmonton, Alta.

Dr Carl H. Heuchert

223 Medical Arts Bldg

Calgary, Alta

Dr Grant K Hoe

11923 Jasper Ave.

Edmonton, Alta

Dr. H M. Hutchuson

10111 138th St.

Edmonton, Alta

Dr. Walter $S$ ohns

512 Rudéau Rc

Calgary, Alta.

Dr Frank L 'Johnson

c/o Campbell Clinic

Lethbridge, Alta.

Dr John H A. Lawrence

1728 26th Ave S W., Apt. 2

Calgary, Alta

Dr A. M. Leacock

Box 910, Olds, Alta.

Dr C M. Learmonth

10322 132nd St.

Edmonton, Alta.

Dr Leopold Lewis

Medicıne Hat Clinic

Medicine Hat, Alta.

Dr F. E. Lundy

3627 12th St. S.W.

Calgary, Alta

Dr Robert I. McCalla

9566 83rd St

Edmonton, Alta

Dr J Alastair Mackay

10344 132nd St.

Edmonton, Alta

Dr Hugh $R$ Macphail

11563 80th Ave.

Edmonton, Alta.

Dr John D. Manes

300 Medical Arts Bldg

Calgary, Alta. 
Dr. A. P Melgrave 1231, Colgrove Ave Calgary, Alta

Dr. D J Mottat 11215 Jasper Ave Edmonton, Alta

Dr G P. Mores 440 Scarboro Ave Calgary, Alta

Dr Walter J. Mudry 632 16th Ave N W Calgary, Alta

Dr W B Murray 601 Madıson Ave Calgary, Alta Dr Harold L N1x 205 McLeod Bldg Edmonton, Alta

Dr N W Nix 10514 134th St Edmonton, Alta

Dr A A O'Nell 609 25th Ave N W Calgary, Alta

Dr Solly G Paletz 11444 71st Ave Edmonton, Alta

Dr L J Patterson Parsons Chinc Red Deer, Alta

Dr Rhoda B Robertson 1839-17th Ave N W Calgary, Alta
Dr John S Ruddell Municipal Hospital Lethbridge, Alta

Dr. Joseph J Scales Dept of Anaesthesia General Hospital Edmonton, Alta

Dr R. D. Scra.gg, 10408 116th Ave Edmonton, Alta.

Dr Noel L. Smith 1404 16th Ave N W Calgary, Alta

Dr Roy R. Spackman Unuversity of Alberta Hospital Edmonton, Alta.

Dr. Adraan W J Starmk Medical Arts Bldg Calgary, Alta

Dr Orest Stechishun

Dept of Anaesthesia General Hospital

Edmonton, Alta

Dr Isabel C M Stevenson 80120 th Ave W

Calgary, Alta

Dr Willam Strome

Harg Clime

Lethbridge, Alta

Dr Peter C Voloshn

11231 75th Ave

Edmonton, Alta

Dr C. Maurice Watson Royal Alexandra Hospital Edmonton, Alta

\section{Saskatchewan Division}

Dr Pamela J Alport

PO Box 523

Regina, Sask

Dr E. Asquith

P O Box 255

Regina, Sask.

Dr M. W Bowenng

P.O Box 34

Regina, Sask.
Dr. Chung Al Chang 1227 15th St.

Saskatoon, Sask

Dr Thomas A Christe 1004 McCallum Hill Bldg P O. Box 34

Regina, Sask

Dr Davina M Cowan 1220 Colony St

Saskatoorı, Sask 
Dr James $F$ Cumming

304 Birks Bldg.

Saskatoon, Sask

Dr. T V. Darke

P.O. Box 125

Regina, Sask

Dr Anthony Roy De Coq Deacock

Dept of Anaesthesia

University Hospital

Saskatoon, Sask

Dr Abram A Dick

521 Bedford Rd

Saskatoon, Sask

Dr Allen B Dobkin

Unıversity Hospital

Saskatoon, Sask.

Dr Harry V Dónaldson

2205 Caurns Ave.

Saskatoon, Sask

Dr D M Ewart

200 Scott Block

Moose Jaw, Sask

Dr Frank C Haley

University Hospital

Saskatoon, Sask

Dr B W Hargarten

107 Medical B.dg

Saskatoon, Sask

Dr John H Harland

Dept of Anesthesia

University Hospital

Saskatoon, Sask.

Dr Ann B. Henschel

1322 Ave K S

Saskatoon, Sask

Dr Albert Hudec

606 Medical \& Dental Bldg

Regina, Sask

Dr Hugh J Johnston

202 Scott Block

Moose Jaw, Sask
Dr. J D. Keene

378 2nd St E

Swift Current, Sask

Dr Christopher J Kılduff

1417 Main St

Saskatoon, Sask

Dr Glen W Kinsman

214 Avenue BIdg

Saskatoon, Sask

Dr Zbıgniew 'T' Kubınsk

1113 Grafton Ave

Moose Jaw, Sask

Dr Violet I A Larsen

Regina General Hospital

Regina, Sask

Dr. D McAlpine

P O Box 34

Regına, Sask

Dr J. E McCutcheon

P O Box 34

Regina, Sask

Dr M Vivyan Morton

908 Saskatchewan Cres E.

Saskatoon, Sask

Dr J E Murphy

PO Box 125

Regına, Sask

Dr Mary/ A Nicholson

141611 the St

Saskatoon, Sask

Dr Otto W Schuh

P O Box 480

Moose Jaw, Sask

Dr W E Upthegrove

735 Unuversity Dr

Saskatoon, Sask.

Dr Gordon M Wyant

522 Copeland Cres

Saskatoon, Sask

\section{Manitoba Division}

Dr Dorothy Barnhouse

283 Belvedere St.

St James, Winnipeg, 12, Man.
Dr Thomas E Beıghton 275 Wuldwood Parc

Fort Garry, Winnupeg, Man 
Dr Marjorie Bennett

St. Boniface Hospital

St. Boniface, Man.

Dr Jack Brener

402 Selkark Ave.

Winnipeg, Man.

Dr Hugh P. Camrass

Dept of Anaesthesia

St Bonface Hospital

St. Bonfface, Man.

Dr. Max Cham

148 McAdam Ave.

Winnipeg 4, Man

Dr Ruchard C. Condon

2N Lanark Gardens Apts.

Lanark St.

Winnipeg, Man

Dr. Willam J. Crawford

295 Carpathia

Winnipeg 9, Man.

Dr. Lionel F. J. Cruckshank

404 Waverly, Ruver Heights

Winnupeg, Man.

Dr Francis Rankin Cullen

Command Medical Office, Ft. Osborne Bks

Winnıpeg, Man.

Dr. John Culhgan

Dept of Anaesthesia

Winnipeg General Hospital

Winnipeg, Man.

Dr. James H Daniels

816 Oxford St

Winnipeg, Man

Dr S. L. Drulak

308 Boreham Blvd

Winnipeg 9, Man.

Dr. Morris Erenberg

784 Ash St.

Winnipeg 9, Man.

Dr. Homer Eshoo

33 Kingston Row

Winnipeg, Man

Dr Myrle A. Gard

812 McDermot Ave.

Winnipeg 3, Man.
Dr. Gordon L. Goulding

Shilo Military Hospital

Camp Shilo, Man

Dr. K. G. Grant

Surte 4, Willis Apts

Norwood, Man.

Dr. J. Hayakawa

967 Winnupeg Ave

Winnege, Man.

Dr Arnold W Holm 1220 Wellington Cres Winnpeg, Man.

Dr. R L Howden

273 Tache Ave

Norwood, Man

Dr D M H Huggins

99 Niagara St.

Wumupeg, Man.

Dr $\mathrm{H}$ ' C' Hutchison

171. Yale Ave.

Winnipeg, Man

Dr. Thorberg Johannesson

763 Renfrew St

Winnipeg. Man

Dr. San Kantor

737 Oac St.

Winnipeg, 9, Man

Dr Elspeth Kent

74 West Gate

Winnipeg, Man

Dr Elızabeth Kolesnıchenko

714 Boyd Bldg.

Portage A.ve

Winnipeg, Man

Di La os Kovacs

408 Se kark Ave

Winnipeg, Man

Dr Ronald S Lambre

Anaesthes1a Dept.

St. Boniface Hospital

Winnipeg, Man

Dr Lours Loban

Ste 24, Jastungs Apts

2] 93 Portage Ave

St James, Man. 
Dr Margaret Loewen

591 Alverstone St.

Winnipeg 10, Man

Dr S M Lugınsky

550 Waverley St.

Winnupeg, Man.

Dr Thomas J. McCaughey

Dept. of Anaesthesia

Chuldren's Hospital

Winnupeg, Man.

Dr Patrick M. F. McGarry

4549 Roblin Blvd.

Varsity View P O , Man

Dr Bernadette P McGivern

Dept of Anaesthesia

Winnipeg General Hospital

Winnipeg, Man

Dr. Robert W. Mulner

169 St Vital Rd.

St Vital, Winnipeg 8, Man

Dr Max Minuck

St Boniface Hospital

St Boniface, Man

Dr Peter Moss

$663 \mathrm{E} \operatorname{lm}$ St.

Winnupeg 9, Man

Dr A. W Natsuk

301 Carpathua Rd.

Winnipeg, Man

Dr D C Askenhead

Burlungton, Ont.

Dr D. W. Aitken

311 Rubidge St.

Peterborough, Ont.

Dr Elizabeth Aitken

147 Sherwood Ave.

London, Ont.

Dr G W. A. Aitken

Box 49

Grand Bend, Ont.

Dr R R. Aitken

147 Sherwood Ave.

London, Ont.
Dr. Peter G. Premachuk

Flin Flon, Man.

Dr B. V Roe

347 Salter St.

Winnipeg, Man.

Dr. Gunther Semelka

546 Cambridge St.

Winnipeg, Man.

Dr Ben Shell

312 Seven Oaks

Winnipeg, Man.

Dr David Tass

395 Elm St.

Winnipeg, Man

Dr G N. Willson

Box 160

Flin Flon, Man

Dr Crustopher Wolkenstein

785 Queenston

Winnipeg 9, Man.

Dr Marcia Wood

711 Brock St

Winnupeg, Man

Dr Wasyl Zaycew

1329 Waller Ave

Winnupeg 9, Man

\section{Ontario Division}

Dr David Allan

De tt. of Anaesthesia

Sic. Chuldren's Hospital

Toronto, Ont.

Dr. C L Anderson

228 King St.

St Catharines, Ont

Dr Damel Antonevych

498 Simcoe St. N., Apt. 14

Oshawa, Ont.

Dr G C. Armitage

PO Box 728

Brampton, Ont.

Dr. Rene Auger

Ottawa Grace Hospital, Wellington St

Ottawa, Ont. 
Dr Eull Nam Aum

Box 510

Bhnd Ruver, Ont

Dr H. A. Axelrod

31 Hilltop Ave.

Toronto, Ont.

Dr Paul Balley 215 Victoria St.

Toronto, Ont.

Dr Zonko Bak

92 Rivercrest Rd.

Tóronto, Ont.

Dr. C. R. Balfour

262 St. Claur Ave. W.

Toronto, Ont.

Dr H. C Balmer,

266 Runnymede Rd.

Toronto, Ont.

Dr H A. Barends

303 Faurmont Ave.

Ottawa, Ont

Dr O R. Bartlett

5020 Riverside Dr.

Riverside, Ont.

Dr Gerald S. Base

298 Woodland Dr

Oakville, Ont.

Dr. D. C. Bastow

30 Rivervew Dr

Toronto, Ont.

Dr E. Bateman

18 Brule Gardens

Toronto, Ont.

Dr R. G. Bateman

217 Pinnacle Ave.

Belleville, Ont.

Dr M. V. Bates

1550 Glen Watson Dr.

Orchard Heights

Port Credit, Ont

Dr Matthew Bazoian

159 Stinson

Hamilton, Ont.

Dr Anita C. Beaton 500 Mountain Park Ave.

Block B, Apt. 202

Hamilton, Ont
Dr. David N. Bell

888 Kukwood Ave, Apt 3

Ottawa, Ont.

Dr Wm H Bentham

98 East Ave. S.

Hamilton, Ont.

Dr D W S Best

2066 Blarrholm Ave.

Burlington, Cint.

Dr G N Black

548 King St.

Port Colborne, Ont

Dr John H Blair

21 King George's Dr

Toronto 9, Ont.

Dr E C. Blatchford

99 Hill Cres

Scarboro, Ont

Dr J A. Blezard

1101 The Parkway

London, Ont

Dr G D M Boddington

167 Rosedale Heights DI

Toronto, Orit

Dr Donald E. Boyer

165 Ballool St., Apt 206

Toronto 7, Ont.

Dr $\mathrm{H}$ W Boyes

417 Rosemary Rd

Toronto, Ont.

Dr J H. L Brennan

68 Fallingbrook Rd

Toronto, Ont

Dr A $\mathrm{T}$ Brown

4 Wychwood Rd

St Cathannes, Ont

Dr G C Bryan

540 Mount Pleasant Rd

Toronto, Ont

Dr W. Butt

7 Conrad Ave

Toronto, Ont.

Dr John R Calder

20 Dundas $S t$

Brantford, Ont

Dr W. S. Caldwell

Box 808 , Brampton, Ont 
Dr Harold Cameron

Dept of Anaesthesia, Westminster Hospital

London, Ont

Dr John D Cameron

55 Kingsmount Blvd

Sudbury, Ont

Dr A D Campbell

165 Queen St N

Kitchener, Ont

Dr Don L Camphell

2769 Lundys Lane

Niagara Falls, Ont

Dr Michael Campbell

Apt 106, 1 Tichester Rd

Toronto 10 , Ont .

Dr S M Campbell

Dept of Anaesthesia, Toronto General Hosp

Toronto, Ont

Dr W A Campbell

123 College $S t$

Kingston, Ont

Dr Joseph T Campolowgo

193 Woolwich St

Guelph, Ont

Dr Doreen Frances Caplun

740 Eglinton Ave W, Apt 507

Toronto, Ont

Dr Joseph Charkoff

9 Faurholme Avenue

Toronto 19, Ont

Dr Margaret Chambers

4 O'Connor Dr

Toronto, Ont

Dr R A. Chaplin

65 Pepler Ave

Toronto, Ont

Dr Peter A Charlébo1s

Apt 203, 484 Church St

Toronto, Ont

Dr John Chassels

14 Deer Park Cres

Toronto, Ont

Dr Kam Chatterjee

39-A Redpath St.

Ottawa, Ont.
Dr George C Clark

892 Avenue Rd.

Toronto, Ont.

Dr Norman S. Clark

110 Milden Hall Rd.

Toronto 12, Ont.

Dr G F. Clement

26-31st St.

Toronto 14, Ont.

Dr Brian H. Coggins

Apt 3,555 Sammon Ave

Toronto, Ont

Dr Arthur H. Cole

142 Wellesley St E., Ajst. 66

Toronto, Ont

Dr Robert H. Cole

531 Highcroft Ave.

Ottawa, Ont.

Dr A E. Conley

75 Pine St. N.

Thorold, Ont

Dr A W. Conn

543 Lakeshore Rd

Mumico, Toronto 14, Ont

Dr Charles P Crompton

71 Valecrest Dr

Toronto, Ont

Dr P W Crookes

Apt 10, 223 The Donway W

Don Mils, Ont

Dr F A Cuddy

201 Bryan St. S

Whitby, Ont

Dr. R K Cumming

22 Lynwood Ave

Ottawa, Ont

Dr E A. Cummings

990 Woodbine Ave

Toronto, Ont

Dr J E Curts

P O Box 463, Fifth Lune W

Streetsville, Ont

Dr. John W Dales

Queensway General Hospital

Toronto 14, Ont 
Dr M. Susan Danels 28 St. Andrews Gdns Toronto, Ont.

Dr. J W. Davidson 15 Limerick Ave Toronto, Ont

Dr. Ethel Dempsey

Box 181

Belleville, Ont

Dr. Frederick T Dennis

187 Arthur St

Port Arthur, Ont

Dr W Derbyshire

70 Princess St

Fort Ene, Ont

Dr H. E. Dovlin

15 Liscombe Rd

Toronto 15, Ont

Dr Jeremiah Dillon

82 Laracor Ave

Oshawa, Ont

Dr G Wesley Dix

117 Laurentide Dr

Don Mills, Ont

Dr. G. D Dixon

92 Inglewood Dr

Toronto, Ont

Dr A. A. Dougan

268 Jedburgh Rd

Toronto, Ont

Dr. Anne Duffield

Nurses' Residence

Wellesley Hospital

Toronto, Ont

Dr J Armand Dufresne

Forbes P O

Ottawa, Ont.

Dr Francis A Duggan

97 Glen Albert Dr

Toronto 6, Ont

Dr. Arthur J. Dunn

20 Rumsey Rd.

Toronto, Ont.

Dr. Donald H Dunn

1189 Queen Victoria Ave

Lorne Park, Ont
Dr. Juhette Durocher

Box 276, R R 1

Ottawa, Ont

Dr W O Moore Ede

44 Warren Rd.

Toronto 7, Ont

Dr. Lewis H Edwards

24 Riverview Dr

Barriefield, Ont

Dr C F. Egan

562 Johnson St

Kingston, Ont

Dr Saul M Eisen

97 Elmsthorpe Ave

Toronito, Ont

Dr Clarence C Evans

147 McNaughton St

Sudbury, Ont

Dr $S$ J Evelyn

377 Vaughan $\mathrm{Rd}$

Toronto, Ont

Dr H Barne Farrley

17 Strathallen Blvd

Toronto 12, Ont

Dr Nicholas Farkas

3945 Tecumseh Rd E

Winclsor, Ont

Dr Anne Marie Fehrmann

485 Mortumer Ave, Apt 6

Toronto 6, Ont

Dr F G C Ferguson

400 Dundas St

London, Ont

Dr R. H Ferguson

315 McMahon Ave

Kingston, Ont.

Dr $P$ W Ferris

40 Brooke Ave

Toronlo, Ont.

Dr E. M. V. Fielding

1105 Valley Way

Niag,ara Falls, Ont.

Dr Shrley Fleming

346 Glengrove Ave W

Toronto, Ont.

Dr. John E. Fox

693 McCowan's Rd

Scarborough, Ont. 
Dr R J Fraser

125 South Oval St

Hamilton, Ont.

Dr Gertrude Freund

31 Alexander St, Apt 1214

Toronto, Ont

Dr J W Fyfe

15I Davd St.

Sudbury, Ont

Dr R A. Gordon

178 St George St.

Toronto 5, Ont.

Dr. J E Gorman

342 Rosedale Blvd.

Windsor, Ont

Dr John E. Green

1319 Laperriere Ave

Ottawa 3, Ont

Dr I. C. M Grumshaw

70 Vaughan $\mathrm{Rd}$

Toronto, Ont

Dr J M Growse

219 Wortley Rd

London, Ont

Dr. T J Halkıewıcz

37 Duncombe Blyd

Scarboro, Toronto, Ont.

Dr Bruce Halliday

Box 249

Tavistock, Ont.

Dr L. M. Hampson

42 Hereford Place

Ottawa, Ont.

Dr. E G. S. Hanley

170 St. George St.

Toronto, Ont.

Dr. T R. Hanley

401 Russell Hull Rd.

Toronto 7, Ont.

Dr Ralph Hargrave

693 McCowan's Rd.

Scarborough, Ont.

Dr Melbourne Harvey

14 Dundana Ave.

Dundas, Ont.
Dr Willam Harvey

463 11th St., "B" West

Owen Sound, Ont.

Dr. Kurt Hellman

25 Forest Ridge Dr

Toronto 10, Ont.

Dr J.S Heron

25 Coxwell Blvd.

Toronto 6, Ont.

Dr. Robert N. Hethernngton

c/o 4 Field Ambulance

Camp Borden, Ont.

Dr N P. Hill

73 Welland Ave

St Cathermes, Ont.

Dr R 'M. Hines

169 Colborne St E.

Oakville, Ont.

Dr R H Holbrook

24 Undermount Ave

Hamilton, Ont.

Dr Gordon M Horne

325 Sammon Ave

Toronto 6, Ont

Dr J H Howell

140 Blair Rd

Galt, Ont.

Dr W. Howitt

115 Woolwich St

Guelph, Ont

Dr E J Jackson

77 Rochester

Toronto, Ont

Dr J R Jacobsen

144 Hilton Ave

Toronto, Ont.

Dr Gesina E. H. Janssen

50 Selkurk St., Apt 409

Ottawa, Ont.

Dr Willam R Johns

39 Wolfe St., Kingston, Ont

Dr. W. S. Johnston

361 Dufferin Ave

London, Ont 
Dr Willam M. Jones

Apt 801, 66 Spadina Rd.

Toronto, Ont

Dr H. T. Jost

267 O'Connor St

Ottawa, Ont.

Dr Brigitte N Kalow

53 Douglas Cres

Toronto 5, Ont

Dr C I. Junkın

100 Onole Parkway, Apt 307

Toronto 7, Ont

Dr F L. Junkın

311 Walmer Rd

Toronto, Ont

Dr Harold T. Kay

22 Sunset Blyd.

Ottawa, Ont.

Dr Hubert B Keenleyside

104 Elworthy Ave

London, Ont

Dr. W Ellott Ketth

2450 Lincoln Rd

Windsor, Ont

Dr M J. Kelly

360 Sixth Ave

Timmıns, Ont.

Dr Robert M Kllborn

174 Lydia St

Kitchener, Ont

Dr Ralph John Kımmerly

152 Simcoe St N

Oshawa, Ont.

Dr F A Kınnarrd

Russell, Ont.

Dr Ebba Kirkebeck

Hospital for Sick Children

Toronto, Ont

Dr R S. Kneeshaw

172 Annette St

Toronto 9, Ont

Dr K. A. Kraft

942 Main St. E

Hamulton, Ont
Dr Maria Kryt

3 Thornton Ave

Toronto 10, Ont

Dr J N Kyles

90 Melrose Ave $S$

Hamilton, Ont

Dr Douglas T Law

Box 567, R R 1

Ottawa, Ont

Dr $\mathrm{H} \mathrm{H}$ Lees

1493 Wyandotte St E

Windsor, Ont

Dr Evan A C. Lloyd

Hospital for S1ck Children

Toronto, Ont

Dr Hubert H Lloyd

34 Beckwith S

Smith Falls, Ont

Dr J R Loudoun

85 Woolwich St

Guelph, Ont

Dr Jeva Rogers Lougheed

561 Broadview Ave

Toronto, Ont

Dr G H. W Lucas

Dept of Pharmacology

Unversity of Toronto

Toronto, Ont

Dr Marıa E Lukowskı

20 Markdale Ave

Toronto 10, Ont

Dr Aht S M lundquist

309 Martland Ave.

Peterborough, Ont.

Dr C W P Lunderville

48 Broac path

Don Milss, Toronto, Ont

Dr Philip C NicCabe

233 Coronada Blvd.

Tecumseh, Ont

Dr A B McCarter

126 Norfolk St.

Guelph, Ont.

Dr Thomas McConnachie

38 Highcluffe Ave.

Hamilton, Ont 
Dr J D C MacDonald

322 Wellington St

Sarma, Ont

Dr John W. McDougall

35 Garden St

Brockville, Ont

Di. Donald W McFarland

P O Box 20

Cookstown, Ont

Dr Wilfred B McGurre

132 Norfolk St S

Simcoe, Ont.

Dr James F MacIntosh

911 Wellington St E

Sault Ste Marie, Ont

Dr Donald $\mathrm{H}$ McIntyre

50 Fraserwood Ave, Apt 2

Toronto, Ont

Dr Iain M MacKay

343 Belsize Dr

Toronto 12, Ont

Dr R G Mackenzie

193 Island Park Dr

Ottawa, Ont

Dr. A M Mackinnon

153A Norfolk St.

Guelph, Ont

Dr Sheilah McLachlin

R R 4, Highland Ave

London, Ont

Dr Kenneth Wilham McLandress

P O Box 320

Mitchell, Ont.

Dr Donald J McLean

107 Simcoe St. S

Oshawa, Ont

Colonel N H. McNally

2060 Woodcrest Rd.

Ottawa, Ont

Dr James H S Mahood

29 Hillcroft Dr.

Kingston, Ont

Dr Maurice Mainville

Box 407, R R 1

Ottawa, Ont
Dr. I. W Mann

$442 \mathrm{~N}$. Christiña St

Sarnia, Ont

Dr Peter Marchant

56 Pıne Cres

Toronto, Ont

Dr. Brran M Masshall

190 Mullwood Rd

Toronto, Ont

Dr J. E Marshall

884 Nora Dr, Long Acres

Burlington, Ont

Dr Alice M Martm

47 Kıngan St

Peterborough, Ont

Dr W E Martun

30 Barbara Cres

Toronto 6, Ont

Dr Donald I Matheson 555 Sammon Ave, Apt ]

Toronto 6, Ont

Dr R L Matthęws

Forest, Ont

Dr Murray Mendelson

259 Searle Ave

Downsvew, Ont

Dr R H Meredith

82 Old Mill Rd

Toronto 18, Ont

Dr Michael W Michalkoff

621 Cummings Ave, Apt 111

Ottawa, Ont

Dr A S. Middlebro

138 Tenth St W

Owen Sound, Ont

Dr D A D Mrlne

Kincardine, Ont

Dr Agnes Moffat

327 Charlotte St

Peterborough, Ont

Dr. Mayhew G. Moore

303 Park St

Kitchener, Ont

Dr. A. G. Morris

536 Wellıngton St

London, Ont. 
Dr. M. L. Morris

118 Simcoe St. N

Oshawa, Ont.

Dr. John K. Moss

32 Tallyho Dr.

Dundas, Ont.

Dr H E Mountain

Chatsworth, Ont.

Dr Gladys Munroe

33 Galt Ave.

Oakville, Ont.

Dr H. A. L. Murphy

2 Grenview Blvd

Toronto, Ont.

Dr James G Murray

14 Highbourne Rd.

Toronto 7, Ont.

Dr H H Nely

Box 70, Cardunal Heights, Ont.

Dr Valentinas A. Nekus

556 Earl St.

Kingston, Ont

Dr. T. R. Nichols

196 Ontano St

Stratford, Ont.

Dr H R. Nicklin

73 Ene St. S.

Leamington, Ont.

Dr Harry T. Norry

3 Mountsfield Dr.

London, Ont.

Dr Rudolph F. Ohlke

2076 Harvest Dr

Port Credit, Ont.

Dr E. A. Oliver

1050 Yonge St, Apt. 37B

Toronto, Ont

Dr. Robert Orange

205 Wembley Dr.

Sudbury, Ont

Dr Kathleen O'Reilly

18 Golfcrest Rd.

Toronto, Ont.

Dr. Stephen J. O'Rourke

110 Sheวpard Ave. E., Apt 201

Willowc ale, Ont.
Dr. T. H. Orton

153 Sumcoe St. IN.

Oshawa, Ont.

Dr Nicholas A. Ost

101 Sear Bldg.

Fort Willam, Ont.

Dr John S Packham

325 Victoria Ave.

Chatham, Ont.

Dr Norman S. Park

282 Bessborough Dr.

Toronto 17, Ont.

Dr. J. A. Peart

33 White St.

St. Thomas, Ont.

Dr P. Arcade Perrier

3.312 Main East

Hawkesbury, Ont.

Dr. V. L. Polit

976 Brucedale Ave. E.

Hamilton, Ont.

Dr J B Poole

417 Dundas St.

Woodstork, Ont.

Dr Ralph I Probert

17 Mount Royal Ave.

Hamilton, Ont.

Dr A. L. Purdon

139 W. Main St.

Welland, Ont.

Dr Ivan Rado

325A James St. S.

Hamilton, Ont.

Dr Edgar Rae

412 Danforth Ave.

Toronto, Ont.

Dr. B. Faxlın

26 Burnside Dr.

Toronto, Ont

Dr Norris H. Ready

Kingston General Hospital

Kingston, Ont.

Dr. W J Rerd

274 Kung St. W

Chatham, Ont.

Dr. J W, Reingwertz

73 Brookview Dr.

Toronto 19, Ont. 
Dr E. E. Reınke

Pembroke, Ont.

Dr W G. Reive

178 Claremont Ave

Kitchener, Ont.

DI Alexander Relle

161 St George St., Apt. 401

Toronto, Ont.

Dr. W A Renwick

119 Duncairn Rd

Don Mills, Ont.

Dr K R Ruchardson

239 Ridout St S.

London, Ont

Dr A. R. Ruddell

84 Homewood Ave.

Kitchener, Ont.

Dr John G. Robinson

42 Greenbrook Dr

Toronto, Ont.

Dr C H. Robson

96 Chatsworth Dr

Toronto, Ont

Dr L E Roche

3 Clemow Ave, Apt. 3

Ottawa, Ont

Dr Edith Rogoman

Wellesley Division, Toronto General Hosp

Toronto, Ont

Dr N. Rosen

327 Palmerston Blvd.

Toronto, Ont

Dr Jean-Paul Rossignol

2030 Harg Dr.

Ottawa, Ont

Dr F Rossitter

186 Wright Ave

Toronto, Ont

Dr Manie Rotenberg

26 Farrington Dr

Willowdale, Ont.

Dr C R Rudolph

118 West Colborne St

Brantford, Ont.
Dr. E S. Russell

$86 \mathrm{McDonnell} \mathrm{St.}$

Kingston, Ont.

Dr. F. G Ruston

44 Beulah Ave

Hamilton, Ont.

Dr P A Sarjeant

674 Broadview Ave.

Toronto, Ont.

Dr Norman A. Scott

311 Rubidge St.

Peterborough, Ont.

Dr B. A Seymour

9 Lytton Blvd.

Toronto, Ont

Dr J M Shapley

189 Dawlish Ave.

Toronto, Ont,

Dr Margaret Sheehan

11 Ranham Place

Don Mills, Ont

Dr Edward J Sheftmán

79 Chelford Rd

Don Mills, Ont

Dr H J Shields

744 Duplex Ave

Toronto, Ont

Dr Henry J Shykoff

1097A Victona Park Ave

Toronto, Ont

Dr Paul Ellss Slater

197 Duchess Ave

London, Ort

Dr Roman L Shuzar

2070 Annapolis Ave

Applewooc Acres

Port Credit, Ont

Dr James Small

306 Kıng St.

Midland, Ont.

Dr Code Smith

20 Heathcote Ave.

Willowdale, Ont.

Dr G. Foster Smith

48 Bennington Heights Dr.

Toronto, Ont. 
Dr. F Van V Snell

66 Binscarth Rd.

Toronto, Ont.

Dr. Wolfgang E. Spoerel

459 Coombs Ave.

London, Ont.

Dr J R Sproule

Ridgeway Clune

Fort William, Ont.

Dr. Natalie M. Staron

58 Greenbrook Dr

Toronto, Ont.

Dr. C. A Stewart

1070 Lombardo Ave

London, Ont.

Dr. Alicja Stralkowska

New Mount Sinaı Hospital

Toronto, Ont

Dr. R M. Stringer

124 Chedoke Ave

Hamilton, Ont

Dr C E. Sylvester

18 Brunswick St

Stratford, Ont

Dr. Tuevo Tenhunen

585 Long Lake $\mathrm{Rd}$

Lockerby, Ont

Dr. Murray C Thompson

7 Windy Rudge Dr

Scarboro, Ont

Dr C E Troping

1 Brentwooc $\mathrm{Rd} \mathrm{N}$

Toronto 18, Ont

Dr Albert A. Toms

3 Bilston Court

Toronto 18, Ont

Dr Michel Tousignant

St Michael's Hospital

Toronto, Ont

Dr N B Urie

420 Hamilton Ave

Ottawa, Ont

Dr. Stuart L Vandewater

65 Fleming Cres

Toronto 17, Ont
Dr J A. Vining

52 Kildeer Cres

Toronto, Ont.

Dr Herbert B Wallıs

290 Wellington

Sarnia, Ont.

Dr G. Owen Warr

138 Kerr St. N.

Oakville, Ont

Dr. D A Warren

221 Jackson St. W

Hamilton, Ont.

Dr A. C Webster

215 Woolwrch St

Guelph, Ont.

Dr W G Webster

31 Southwick St.

St Thomas, Ont

Dr $\mathrm{R}$ Leonard Weston

105 Anndale Dr.

Willowdale, Ont

Dr - S. Whalen

2 Ec.gehill Rd

Toronto, Ont.

Dr D W Whyte

360 Huron Ave

Ottawa, Ont

Dr A R, Wilkins

170 St George St.

Toronto, Ont.

Dr Marta-Maret Wilo

406 Castlefield Ave

Toronto 12, Ont.

Dr V L Wincott

305 Sarah St, Box 400

Chippawa, Ont

Dr Jules Winemaker

3590 Church St

Windsor, Orit

Dr $\mathrm{H}$. Winesanker

459 Bloor St W.

Toronto 4, Ont

Dr J M Wishart

311 Rubidge St

Peterborough, Ont

Dr G C Wray

15 London St. S

Hamilton, Ont. 
Dr H A Wrong

2180 Main St.

Niagara Falls, Ont.

Dr. Felix Wuerth

Victoria Hospital

London, Ont

Dr Vasil Y. Yancoff

20 Warland Ave.

Toronto 6, Ont

Dr Eugene Allard

1583 St Cyrille W

Quebec, Que

Dr Marc Allen

1005 St Prospère.

Trois Rivières, Que.

Dr Wills M. Archibald

5210 Mariette Ave

Cote St Luc

Montreal, Que

Dr ean Archambault

349e Mountain St.

Montreal, Que

Dr J W Armstrong

5010 MacDonald Ave., \#17

Montreal 29, Que

Dr Ubald Aubry.

3206 Sherbrooke Est

Montreal, Que

Dr Joseph Aubut

1694 1st Ave.

Quebec, Que

Dr J E Barbeau

700 Cremazie est

Montreal, Que

Dr. A Beaudet

1816 Blvd Laurd

Mount Royal, Que.

Dr Jazeps $\mathrm{T}$ Beldavs

6735 Lemay St.

Rosemont, Montreal, Que.

Dr. Léo Benoit

585 Ave. Mondor

Ste Hyacinthe, Que.
Dr Ming Yao

35 Fern Ave

Ottawa, Ont

Dr John M Yoshicka

369 Morrison Rd

Oakville, Ont

Dr. B. G Zrelonka

2 Fenley Dr. (Group Box 162).

Toronto 15, Ont.

\section{QueBec Drvision}

Dr Jean-Maurice Bergeron

12625 th Ave

Shaw1ngan Sud

Montreal, Que

Dr Jogues Berthiaume

Hôpital Hôtel-Dieu

3840 St Urbain

Montreal, Que

Dr Paulne Biron-Beaudry

1462 E St Joseph Blvd., Apt 4

Montreal, Que

Dr A Borsvert

Box 727

Rouyn, Que

Dr Jean. Lours Bosvin

2967 de Francheville

Trois-Rivières, Que

Dr A Boni 1

4074 Blvd _asalle

Verdun, Que

Dr Robert $R$ Boright

4068 Grey Ave.

Montreal 28, Que

Dr Julita L. Borzecki

4910 Walkley Ave

Montreal, Que.

Dr Marcel A. Boudreau

203 St Jean Baptiste

Rimouski, Que

Dr. Wesley Bourne

2165 Lincoln Ave, Api 18

Montreal 25, Que

Dr Helen Cooper Bowman

266 St Laurent

Rosemere, Que. 
Dr. J. Maurice Boyer

70 Champlain

Valleyfield, Que.

Dr Victor Brassard

501 8ième Ave.

Grand'Mère, Que

Dr G F Brondle

3644 Ontano Ave, Apt 62

Montreal, Que

Dr Philup R. Bromage

24 Fieldfair Ave

Beaureparre, Que

Dr Jean-Lous Brunet

6560 Blvd. Pre IX

Montreal, Que.

Dr Adol she Byk

Hôpital c u Sacre-Cour

3300 Ouest Blvd. Goum

Montreal, Que

Dr Gaston Cangnan

10595 . Hamel

Montreal 12, Que

Dr. M. C Cheng

5615 Palmer Ave

Côte St. Luc

Montreal, Que.

Dr. Georges A. Chevrette

554 Blvd Manseau

Joliette, Que.

Dr Jean Choulnard

485 Champfleury

Quebec, Que

Dr Miodag Cirkovic

2620 Rue Bellechasse

Montreal, Que

Dr Moise Clermont 3511 Ave Grrouard

Montreal, Que

Dr G. A. Cloutier

7416 Christophe-Colomb

Montreal, Que.

Dr. Max Cohen

4947 Lacombe Ave

Montreal 26, Que.

Dr Gaston Comtois

190 Blvd. St. Sacrement

Chicoutim1, Que
Dr Branislava M. Cop-Hodjera

3165 Linton Ave., Apt. 3

Montreal, Que.

Dr George Courchesne

1280 rue de Callières

Quebec, Que.

Dr George Cousineau

5700 Gouln Blvd W

Montreal, Que

Dr IV G Cullen

226 Ballantyne Ave $\mathrm{N}$

Montreal, Que

Dr Lous V Cullınan

1782 College St

St Laurent,

Montreal 9, Que

Dr John K. H Culverhouse

5275 rue de Bellechasse, Apt 5

Montreal 36, Que

Dr Egbert Dangle

1346 Rue Garn'er

Quebec, Que

Dr H T Davenport

Montreal Chuldren's Hospital

Montreal, Que

Dr P Decary

$2852 \mathrm{St} /$ Joseph Blvd E

Montreal, Que

Dr Jean-Paul Dechêne

28 ayenue de la Colline

Ste Foy, Qe.

Dr Rosario Denis

5.300 Blvd Pre IX

Montreal, Que

Dr Hector Desrosiers

2174 Centre St.

Montreal, Q̧ue.

Dr Simon Dombrowsk

1600 3rd Ave.

Quebec, Que.

Dr Henrı Drolet

211 ouest Saint-Cyrille

Quebec, Que

Dr. Marius Dubeau

2060 Graham Blvd.

Montreal 18, Que. 
Dr Antomo Duchesne

3.3A Rue Centre

Cranby, Que

Dr Roland Duchesne

1102 Demontigny St

Quebec, Que

Dr Yves Dugas

10453 Hamel

Montreal, Que

Dr Francis A Duggan

97 Glen Albert Dr

Toronto 6, Ont

Dr Henrı Durand

16 6th Ave

Charlesbourg, Que.

Dr Claude Duval

2510 Bellechasse

Montreal, Que.

Di Gladys Ellison 3460 Simpson St.

Montreal, Que

Dr Rohert Ferguson

739 Upper Belmont Ave

Westmount, Que

Dr John D A Furth

Apt 33, 3644 Ontano Ave

Montreal, Que

Dr Guy Fortm

69 Richelıeu Blvd

St Hilare, Que

Dr Violet E. Frost

492 Flm Ave

Montreal 6, Que

Dr A Gagliano

9250 Lumșden St

Montreal 11, Que

Dr A Amé Gagné

10 McMahon, \#24

Quebec, Que

Dr Roger Gagnon

504 Blvd. des Prairies

Laval des Rapides

Montreal 9, Que

Dr Paul Galibors

355 221ème Rue

Quebec, Que
Dr James M B Garland

Montreal General Hospital

Montreal 25, Que

Dr Jean-Françoıs ,Garneau

5300 Rue Beaubien Esı, Apt. 9

Vontreal 36, Que

Dr L H Gatıen

3810 St Joseph Blvd

Lachine, Que

Dr Philispe Gẹdron

St Josep i Hospital

Three Rivers, Que

Dr Roger Gervais

5590 Ave Victona

Montreal, Que

Dr Gaston Giguère

St-Michel

Comté de Bellechasse, Que

Dr Paul-Emule Gilberı

Hòtel-Dieu, St Jerome

Cté Terrebonne, Qué

Dr R G Glbert

Montreal Neurological Institute

Yontreal, Que

Dr Dardre M M Gillies

6607 Côte St Luc Rd, Apt 310

Montreal, Que

Dr W N Godin

Three Rivers, Que

Mr Marcel Goyer

259 Maisán

Drummondville, Que

Dr H R Gruffith

3445 Northcliffe Ave

Montreal, Que

Dr Elzear Hamel

3672 Blvd Lasalle

Verdun, Que

Di Albert Hamelin

197 de la Ronde

Quebec, Que

Dr Paul Hameln

4800 Blvd P1e 1X, Apt. 21

Montreal, Que. 
Dr Ian Harper

Dept. of Anaesthesia

Montreal General Hospital

Montreal, Que

Dr Claude D Hébert

352 16th St.

Quebec, Que.

Dr M. Hoffman-Zukowskı 7770 Stuart Ave

Montreal, Que.

Dr. Jacques Houde

Hôtel-Dieu de Quebec

Quebec, Que.

Dr. Roland Hould

2750 Montarville

Quebec 10, Que

Dr Fernando Hudon

1601 Blvd, Laurier

Sillery, Que.

Dr André Jacques

Dept of Anaesthesia

Hôtel-Dieu de Québec

Palace Hill, Quebec, Que.

Dr Alfred Jobidon

2352 Choquette

Quebec, Que.

Dr. Raymond Joly

340 Labelle

St. Jerome, Que.

Dr Jakob V. Kalnins

3015 Barclay Ave., Apt. 7

Montreal, Que.

Dr. George S. Kambourıan

Montreal General Hospital

Montreal, Que.

Dr. Jacques Houde

Hôtel-Dieu dé Quebec

Quebec, Que

Dr. Roland Hould

2750 Montarville

Quebec 10, Que

Dr. Fernando Hudon

1601 Blvd., Laurier

Sillery, Que

Dr André Jacques

Dept of Anaesthesia

Hôtel-Dieu de Québec.

Palace Hill, Quebec, Que.
Dr. Alfred Jobidon

2352 Choquette

Quebec, Que.

Dr. Raymond Joly

340 Labelle

St. Jerome, Que.

Dr Jakob V. Kalnins

3015 Barclay Ave, Apt. 7

Montreal, Que.

Dr Georgr S Kambourian

Notre Dame Hospital

Montreal, Que.

Dr Mıcháel Keéri-Szanto

704 Kanora Rd.

Mount Royal Montreal, Que

Dr J J Kelly

423 Roslyn Ave.

Westmount, Montreal 6, Que

Dr Dand Korman

5585 Clanranald

Montreal, Que

Dr. Jeannine L Ladouceur

3154 Fendall

Montreal, Que

Pr. Jules Lafleur

Lachute, Que.

Dr. Gerrard Lafortune

7312 Papineau

Montreal, Que.

Dr. Joseph Lafortune

422 Manseau Blvd.

Joliette, Que.

Dr Raymond L'Allier

8230 Chamagneur

Montreal 15, Que.

Dr. Yves Lamarche

816 Panchaud St.

Quebec, Que.

Dr Georges Lambert

1027 Commerciale

St Romuald, Que.

Dr J J. Larnbert

475 Wiseman Ave, Outremont

Montreal 8, Que.

Dr. Lours Lamoureux

930 West Gouin Blvd.

Montreal, Que. 
Dr Rouville Lamy 191 Sud Rue Brooks Sherbrooke, Que.

Dr. Jean Langevin 493 Mitchell Ville Mount Royal, Que.

Dr. Irenne Lapierre 1213 Commerciále St Romuald, Que.

Dr. Jean Laporte 10441 Hamel Montreal, Que.

Dr. Eric W. Larking Montreal General Hospital Montreal, Que

Dr Patrick F. Laughrea

515 Fecteau

Thetford Mines, Que

Dr Jean Paul Lavellée 1679 Charles Falkner Ahuntsic, Montreal, Que

Dr Joseph Leavitt 4489 Maplewood Ave. Montreal, Que

Dr René Lebeau 3725 Maplewood Montreal, Que.

Dr. J. Jacques LeBlanc 27 Gendron St Hull, Que.

Dr Claude Leduc 803 de Fleurimont Montreal 10, Que.

Dr Lionel J Leduc 2160 Capitaune Beriner Bordeaux, Montreal, Que.

Dr. Maunce Legare 2171 Sherbrooke St. E Montreal, Que.

Dr L. Legault 300 Carré St. Louis Montreal 18, Que.

Dr Richard Lemieux Buckingham, Que
Dr René Létienne

Clinque Medicale du Parc Lafơntaipe 1657 Rue du Parc Lafontaine Montreal 34, Que.

Dr. Marcel L'Etoile 6417 8th Ave.

Rosemont, Montreal, Que

Dr. Armand Levac 4395 Ave. Verdun Montreal, Que.

Dr G A. Lew1s 12 Lakeshore Rd. Beáconsfield, Que.

Dr. Leon Longtin 45 Hazelwood Outrement, Que.

Dr Edouard Lorhe 6531 Molson St Montreal, Que

Dr R. Lussier 3814 Grey, N. D G. Montreal, Que.

Dr Alexander W. McDonald 4665 Prince of Wales Ave Montreal 28, Que.

Dr Robert Macdonald Apt 104, 5304 Macdonald Ave Côte St. Luc Montreal, Que.

Dr P McLaughlon 7045 St Denis St Montreal, Que

Dr P. McMahon 166 King St. W. Sherbrooke, Que.

Dr D. W. MacMillan 1165 Maple Ave. Quebec 6, Que.

Dr. David J L MacWatt 7375 Maynard Mount Royal, Montreal, Que

Dr. Maria Majchrowicz 3511 Shuter St., Apt. 6 Montreal, Que.

Dr Paul Marcoux

Hôtel -Dieu de St. Jerome

St. Jerome, Co Terrebonne, Que 
Dr R L Marın

127 Councl

Sherbrooke, Que

Dr A Marsolas

1251 Rue St Dems

Montreal, Que

Dr Emıle Martmbedu

3165 Rue Trembliy,

Montreal, Que

Dr Victorin Masson

137 St Domınıque

Ionquière, Que.

Dr Lucien Mercier

415 Ste Hélène

Montreal Sud, Que

Dr Jean-Paul Methot

12260 Blvd. Taylor

Montreal, Que

Dr Gerard G Mignault

166 Fleury St. E

Montreal, Que

Dr Velta K Mikelsons

3768 Botrel Ave.

Montreal, Que.

Dr Rosaire Milette

200 Ste Damasse

Drummondville, Que

Dr Ronald A. Millar

Dept of Anaesthesia

Montreal Neurological Institute

Montreal, Que

Dr D A. Mondor

4902 Verdun Ave

Verdun, Que

Dr. La Salle Mondor

630 6ième Rue

Grand'Mere, Que

Dr. William W Montın

1125 Monctea Ave

Quebec, Que

Dr Yvon Monnville

Louseville, Que

Dr. John $S$ Nielson

5255 Dudemaine St, Apt. 5

Ville St Laurent

Montreal 19, Que
Dr. A B Noble

316 Lethbridge Ave

Mount Royal, Que

Dr. J Ovila Noel

301 Councl

Sherbrooke, Que

Dr Ralph R Novon

Notre Dame Hospital

Montreal, Que

Dr Peter E O'Shaughnessy

3198 Lacombe

Montreal 26, Que

Dr Paul E Otton

11590 Lavigne St

Montreal 9, Que

Dr Paul Ovellet

2095 lère Ave

Quebec, Que.

Dr B Paiment

666410 th Ave

Rosemount, Que

Dr Pierre Paquet

259 D'Aigullon

Quebec, Que

Dr Bermand Paracis

2110 Bourbonnère

Quebec, Que

Dr Wilfrid Pellerm

I76 Rue Sherbrooke

Magog, Que

Dr Jean-Gruy Patome

166 St Françols Est

Quebec, Que

Dr Jacques Pelletier

853 Ave Panchand

Quebec, Que

Dr Loyola Perras

530 Grosvenor

Westmount, Que

Dr Luc Perreault

8493 St Laurent

Montreal, Que

Dr Paul llerron

3075 Maplewood, Apt 7

Montreal 26, Que. 
Dr Anthony Petrasek

Dept. of Anesthesia

Queen Mary Veterans Hospital

Queen Mary Rd

Montreal, Que

Dr Jean Phaneuf

2748 Rue Girouard

St Hyacinthe, Que

$D_{1}$ Jean-Guy Phaneuf

228 Rue Longurel

St Jean, Que.

Dr Gilles Piche

Arthabaska, Que.

Dr H Pilon

251 St Joseph Blid

Hull, Que.

Dr Marcel Plamondon

820 Des Braves Ave

Quebec, Que

Dr Gabriel Plante

46 Ave Lamontagne

Quebec, Que

Dr Paul Plourde

Hospital Hôtel Dieu

Montmagny, Que

Dr Roger Poirici

231 Longuel

st lean d'Iberville, Que

Dr Ph Poliquin

Manıwakı

Co Gatmeau, Que

Dr I Claude Poulot

1214 Maxfield

Quebec, Que

Dr. Dand J Power

4131 Harvard Ave, N D.G

Montreal, Que

Dr Hortense Pruḍ'homme

131 Graham Blvd

Montreal, Que

Dr Paul Quan

Montreal Children's Hospital

2300 Tu>per St

Montrea 25, Que

Dr J A Reny

2553 Beaubien St. E.

Montreal, Que
Dr Margaret A Rensad

Montreal Neturological Institute

Montreal, Que

Dr Anne B Ruchardion

Anzesthetic Dept

Royal Victoria Hospital

Montreal, Que

Dı Helmut A Ruchteı

Arvida, Que

Dr Lucien Rintret

1045 Ave Begm

Quebec 6, Que

Dr André Rioux

4572 Wellington

Verdun, Montreal, Que

Dr James M G Ritche

Royal Victoria Hospital

Montreal, Que

Dr Francis A Robillard

Vontreal General Hospital

Montreal, Que

Dr I Maturice Robillard

4000 Ave Marlowe

Montreal 28, Que

Dr I Gordon Robson

301 Victoria Di.

Bule d'Urfée, Que

Ds féch $\mathrm{L}$ Rochetort

Jean Talon Hospital

Montreal, Que

Dr José Kos Rosales

7062 Chester Ave, N D G

Montreal, Que

Di J M Roulédu

St Grégoure $\mathrm{Ca}$

Nicolet, Que

Dr Lucien Rousseau

642 Bloomfield, Outremont

Montreal, Que

Dr C A Rous

131 North St Charles

Joliette, Que

Dr C E Roy

Hòpital St Luc 1058 St Dens

Montreal, Que

Dr. D. Saindon

C P 116 Rivière-du-Loup, Que 
Dr. Charles A. Shendan

17 Highfield

Mount Royal, Que.

Dr. Fernand Shooner Hospital Notre Dame Montreal, Que.

Dr. Gaston Simard 130 Champlain Bare Comeau, Que

Dr. A. V. Sumon 4865 Queen Mary Rd Montreal, Que.

Dr, Paul E. Sirois 129 Claure-Fontaine Quebec, Que.

Dr. B. S. Skanner

Apt 4, 2203 St. Catherine St W Montreal, Que.

Dr. H. M. Slater

5045 Prince of Wales Ave.

Montreal, Que.

Dr. H. G. Smith

18B 5th St.

Shawinigan Falls, Que.

Dr. John Arnold Smith

Dept of Anaesthesia

Montreal General Hospital

Montreal, Que.

Dr. Y. Sormany

1031 Desaulniers

Chicoutimi, Que

Dr. Hildegard Strathmann

Royal Victoria Hospital

Montreal, Que.

Dr. John B. I. Sutherland

$450 \mathrm{Elm}$ Ave.

Westmount 6, Que.

Dr. F. Tanquay

100 Murray

Sherbrooke, Que.

Dr. C. W. Tanton

104 Thurlow Rd.

Hampstead, Que.
Dr. Roger J Thompsion

114 Forest Gardens

Pointe Claire, Que

Dr. A. Tíltıns

Hôputal Sante-Justirie pour les Enfants

Montreal, Que

Dr Lionel Tremblay

2752 Blvd Rosemont

Montreal, Que

Dr W Tremblay

10612 Blvd Auteul

Montreal, Que

Dr. Hugues Turner

5630 Ave. Decelles, Apt 5

Montreal, Que.

Dr J W. K Tyl

3440 Ridgewood Ave , Apt. 44

Montreal 26, Que

Dr Annegret Uhthoff

498-38th Ave, Ville Lasalle

Montreal, Que

Major G Alan VanVhet

Lacolle, Que

Dr. Row'éo Velleux

208 King St. E

Sherbrooke, Que

Dr Jean Vigneau

230 Mercille Ave

St. Lambert, Que

Dr Pat Vigneault

4340 Pie 1X, Apt 1

Montreal, Que

Dr Dagmar Vosu

Catherine Booth Maternity Hospital

440 Walkley

Montreal, Que.

Dr. W. A. Wielhorska

Maisonneuve Hospstal

Montreal, Que.

Dr. F. A H. Wilkirison

406 Pine Ave. W.

Montreal, Que.

Dr. John E Wynands

7022 Somerled Ave

Montreal, Que 


\section{New Brunswick Division}

Dr L. E. Belanger

P O Box 296, Edmunston, N B

Dr A M R Brown

15 Coburg St.

St John, N.B.

Dr Jean Caron

416 Golf St

Bathurst, N.B

Dr Adeline E. Comeau

Hôtel Dieu Hospital

Bathurst, N.B.

Dr. F. R. Connell

177 Princess St.

St. John, N.B.

Dr. J. Daigle

Dalhousie, N.B.

Dr E. R. Davis

42 Coburg St.

St John, N.B.

Dr Joseph A. Dobson

521 Blythwood Ave.

Riverview Hts., N.B.

Dr. Lewis $\mathrm{H}$ Freedman

119 Prncess St.

St John, N B

Dr. Roger Guerrette

Tracadie, N.B

Dr. F C. Jennıngs

38 Paddock St.

St John, N.B.

Dr. C. Graham Kee

126 Elm St.

Woodstock, N.B
Dr Stewart T. Love

361 Regent St.

Fredericton, N.B.

Dr. E. W. Lunney

42 Coburg St.

St. John, N.B.

Dr. Thomas E. Lunney

42 Cobourg St.

St John, N.B.

Dr J A. MacDóugall

8 Queen Square.

Saint John, N.B.

Dr George W. McElman

69 Bridge St., Fredericton, N B.

Dr. - . Fs McInerney

361 Regent St.

Fredericton, N.B.

Dr. William A. Oatway

266 Cameron St

Moncton, N.B.

Dr. G. V Parsons

78 Henry St.

Moncton, N.B.

Dr. Ralph L. Prime

361 Regent St.

Fredericton, N.B

Dr. Louss Rustige

104 Leinster St

St John, N.B.

Dr Matthew $\mathrm{H}$ Swan

78 Rosebery $\mathrm{St}$.

Campbellton, N.B

\section{Nova Scotia Division}

Dr Thomas A. Anderson

3 Burns Dr.

Armdale, Halifax Co., N S.

Dr C. H. L. Baker

80 Connaught Ave.

Halifax, N S.

Dr $R$ W. Ballem

29 Doug Smith Dr.

Hainfax, N.S.
Dr. Stephen B Bird

Liverpool, N S

Dr. Peter Blake-Knox

Hammonds Plains Rd.

Bedford, Halifax Co., N.S.

Dr Charles W. Carr

Camp Hill Hospital

Halifax, N.S. 
Dr George Donaldson

Mahone Bay, Lunenb Co, N S

Dr Stanislaw B Donglewicz

P.O Box 638

Antigonish, N S

Dr D Drury

156 Victoria St

Amherst, N S

Dr. R A Fleming

3 Crowsnest Dr

Halifax, NS

Dr Ross B Irwin

71 Lousburg Lane

Dartmouth, N.S

Dr. Masatatsu Ishn

130 Willow St

Halıfax, N S

Dr. A S Macintosh

313 Spring Garden Rd

Halıfax, N.S
Dr E J. MacLeod

8 Glenwood Ave

Dartmouth, N S

Dr Roberta Nichols

248 Tower Rd.

Halıfax, N.S.

Di A F Pasquet

198 Inglis St

Halifas, N S

Dr. Hadsel $\mathrm{G}$ Quigley

37 Bellevue Ave

Halifax, NS

Di Gordon Simpson

26 Rigby $\mathrm{Rd}$

Sydney, N\&

Dr C C Sioddard

207 Coburg Rd

Hallda, N\}

Dr Andrew $S$ Wenning

16 Chain Rock Dr

Hahfax, N s

\title{
Newfoundland Division
}

Dr Raymond L Bugden

38 Brookfield Ave

Corner Brook, Nfld

Dr. Peter W Edward

Box 343

Corner Brook, Nfld

Dr. Charles U. Henderson

Waterford Bndge Rd

St. John's Nfld

Dr. C D Kean

17 Topsall Rd

St. John's Nfld

Dr. John James Kennedy

188 LeMarchant Rd

St. John's, Nfld

\author{
Dr Abraham Levitz \\ 140 Elizabeth Ave \\ St John's Nfld \\ Dr William David Parsons \\ 95 Long's Hill \\ St John's, Nfld \\ Dr Hazel N Peisley \\ Apt 1, Churchll Square Apts \\ St John's NAld \\ Dr Edward L Sharpe \\ Grand Falls Clinic, Grand Falls, NAld \\ Dr. Alan W Simpson \\ General Hospital, St John's, Nfld \\ Dr Thomas G Stentaford \\ 11 Winter Ave \\ St John's, NHd
}

\section{Prince Edward Island Drvision}

Dr. C. M Dewar

O'Leary, P E I

Dr. Gilbert J. Gallant

281 Schurman Ave

Summerside, P.E.I
Dr L E Prowse

29 Greenfield Ave

Charlottetown, P E I 


\section{U.S.A.}

Dr Geordis M Aasherm

Northern State Hospital

Sedro Woolley, Wash.

Dr A K Bradshaw

2522 65th Place $S$ E

Mercer Island, Wash

Dr John I. Daves

Dept of Anaesthessa

Unversity of Kansas Medical Center

Ranbow Blvd at 39th St.

Kansas City 12, Kan

Dr J E Dupuy

2554 East Blvd,

Cleveland 4, Ohıo

Dr Lionel Glassman

6002 Wallss Ave

Baltımore 15, Md

Dr Ian S H Harper

Apt C, 4534 North Charles St

Baltumore 10, Md

Dr J E Higgins

214 Notre Dame St.

Joliet, Ill

Dr Eric B Howell

12345 Cedar Rd

Cleveland Heights, Ohıo

Dr. Wm P Jones

Cleveland Clinic

2020 East 93rd St

Cleveland 6, Ohıo

Dr Marvin Kay

15-H Hunt Walk

Bergenfield, N. J

Dr C M Kincarde

Lahey Clinic

Boston, Mass

Dr Hannaliese Kralemann

Dept. of Anaesthesia

University Hospital

Minneapolıs, Minn.
Dr Gerard R, F, Landry

216 Grace St.

Councl Bluffs, Iowa

Dr Gerard Lavole

Duke Hospital Medical Center

Division of Anesthesiology

Durham, N.C.

Dr Joseph McCammon

21220 th Ave. S. W

Rochester, Minn

Dr. Cyril R MacLean

817 Waldo Blvd.

Mantowoc, Wis

Dr. John F MacLellan

"Orchard Hull"

Rte 3, Valley Pike

Winchester, $\mathrm{Va}$

Dr E Maroulakis

7742 South Cornell Ave.

Chicago 49, Ill.

Dr. Elızabeth M Martin

3 Ruver Rudge Rd

Hudson, N.Y.

Dr Horacio Martinez

Dept of Anaesthesia

Unversity Hospitals

Western Reserve University

Cleveland, Ohio

Dr Gerda S Menetrez

5231 Chevy Chase Parkway, N.W.

Washington 15, D.C.

Dr Emerson A Moffit

1218 6th St S W.

Rochester, Minn.

Dr. Arthur J. Oswald

University of Minnesota Hospital

Room C 596

412 Unıon St. S E.

Munineapolis, Minn.

Dr Edward Petrovich

Henry Ford Hospital

Farndale, Mich. 
Dr Jean Quntal

Campus, Apt. 15

Elf St.

Durham, N C.

Dr. R Rabbitt

Variety Children's Hospital

Miami, Fla.

Dr Imbi Ralyea

16645 Freeland

Detroit 35, Mich.

Dr Norbert Schnelle

Mayo Clinic

Rochester, Minn.

Dr William A Seibert

4535 Zion St.

Little Neck, Long Island 62, N Y
Dr C R Stephen

Division of Anaesthesiology

Duke Unuversity

Durham, N.C.

Dr Thomas N F Todd

2825 Benton Blvd.

Minneapolıs, Minn

Dr Arthur Torrie

905 E Columbia

Seattle 22, Wash

Dr E H. Watts

P.O. Box 996

Scottsdale, Artz.

Dr James K Whittal

703 Bellvue N

Bellroy Apartments

Seattle, Wash

\section{Other Countraes}

Dr. M. Mufit Erkul

1 incl Cerrahı KTinigi

Gerrahpasa Hastahanes1

Arksaray, Istanbul, Turkey

Dr Gordon D Glennie

"Watergate"

Wadhurst, E Sussex, England

Dr Helen E Gordon

7 Well Rd, Hampstead

London N.W. 3, England

Dr C Langton Hewer

33 Stormont Rd Highgate

London N 6, England

Mr A Charles King

27 Devonshire St

London W.1, England

Dr Kwang y1 Lee

Anesthesia Dept.

National Tawwan University Hospital

Tauper, Taiwan (Formosa), Chuna

Professor Sur Robert Macintosh

Pembroke College

Oxford, England

Dr. Sheila Murphy

Woodview, West End

Castletownbere

Co. Cork, Ireland
Dr Geoffrey Organe

17 Burghley Rd, Wimbledon

London, S W 19, England

Dr Mukteshwar Prosad

Darbhange Palace Rd

Chouhatta, Patna 4

Bihar, India

Dr Victor A Rogyers

c/o Canadian Government

34 Mooresfield

Liverpool 2, England

Dr W N Rollason

83 Newland Park

Hull, Yorkshire, England

Dr Helen M Rossiter

c/o Prof R J. Rossiter, Biochemistry

John Curtain School of Medicine

Austrahian Natıonal Unıversity

Canberra, Australia

Dr. Willıam D A Smith

The Royal South Hants Hospital

Southampton, Hants, England

Dr Edward T. Thomas

Hullingdon Hos jital

Uxbridge, Midclesex, England

Dr A C Yelland

3 (F) Wing R.C A F

CAPO 50.55

Canadian Armed Forces, Europe 